\title{
Deniable Encryption Key
}

\author{
P.Lokesh Kumar Reddy ${ }^{1}$, B.Rama Bhupal Reddy ${ }^{2}$, S.Rama Krishna ${ }^{3}$ \\ ${ }^{I}$ Rama Raja Institute of Technology and Science, Tirupati, A.P., India \\ ${ }^{2}$ Dept. of Mathematics, K.S.R.M. College of Engineering, Kadapa, A.P., India \\ ${ }^{3}$ Dept. of Computer Science, S.V. University, Tirupati, A.P., India
}

\begin{abstract}
Deniable encryption is an important that allows a user (a sender and/or a receiver) to escape a coercion attempted by a coercive adversary. Such an adversary approaches the coerced user after transmission forcing him to reveal all his random inputs used during encryption or decryption. Since traditional encryption schemes commit the user to his random inputs, the user is forced to reveal the true values of all his random inputs (including the encrypted/decrypted messages and the encryption/decryption keys) which are verifiable by this coercer using the intercepted cipher text. In this scenario, a coercer may force the user to perform actions against his wish. An appealing property in the mediated RSA, PKI was introduced that, the user has no information, neither about his full private (decryption) key, nor the factorization of the RSA public modulus, which represents an excellent step toward achieving in forcibility in public key encryption, since, a coercer cannot ask the user to reveal such unknown information. In this pa-per we present a scheme for receiver-deniable public-key encryption, by which, the receiver is able to lie about the decrypted message to a coercer and hence, escape a coercion. On one hand, the receiver is able to decrypt for the correct message, on the other hand, all the information held by the receiver, when opened to a coercer, do not allow this coercer to verify the encrypted message and consequently, approaching this user becomes useless from the very beginning.
\end{abstract}

Keywords: Deniable encryption, mediated PKI, oblivious transfer, public-key encryption, RSA

\section{Introduction}

While traditional encryption schemes protect the privacy of the sender and the receiver against eavesdroppers (semantic security), they fail to provide protection against coercers. A coercive adversary has the power to approach the user (the sender and/or the receiver) after the ciphertext is transmitted and of course recorded by this adversary. It commands the user to reveal all his random inputs used during encryption or decryption. Since the cipher text produced, using standard encryption schemes (specially, publickey encryption) commits the user to his random inputs, It cannot lie about the true plaintext. Such commitments allow the coercive adversary to verify the validity of the opened message. Deniable encryption allows a user to escape coercion. Namely, if this user opens all his inputs (including the claimed encrypted message) to a coercer, the coercer fails to prove the validity or invalidity of the opened message.

Deniable encryption maybe classified according to which party is coerced: sender-deniable encryption schemes are resilient against coercing the sender. The Definitions for receiver-deniable and senderreceiver- deniable follow analogously. When the sender and the receiver initially share a common secret key, this is spoken off as shared-key deniable encryption. In deniable public- key encryption, no pre-shared information and no communications are assumed prior to the encryption process. This follows from the assumptions of standard public-key encryption schemes. Yet, deniable public-key encryption is more challenging than deniable shared-key encryption since the public key of the receiver is already known to everyone including the coercer, consequently, neither the sender nor the receiver can lie about the receiver's public key.

The work in [5] showed that it is possible by simple tricks to transform any sender-deniable encryption scheme to a receiver-deniable encryption scheme and vice- versa. Also, they showed that, with the help of other parties with at least one of them remains un-attacked, it is possible to transform a senderdeniable encryption scheme to a sender-receiver-deniable encryption scheme.

In our recent work of, we devised a sender-deniable public-key encryption based on quadratic residuosity of a composite modulus and showed how to device a sender- deniable public-key encryption from any trapdoor per mutation. However, when the schemes are transformed to be receiver-deniable using the tricks of [5], the schemes are no more one-move schemes. Considering only one- move schemes, receiver deniability is more challenging than sender-deniability since in the later case, everyone knows the public-key of the receiver but the private key of the receiver is known only to the receiver who is beyond the reach of the coercer. In the former case, the receiver maybe coerced to reveal his private key which is verifiable using the public key and a dummy message. 
Deniable encryption is very useful in the protocols where coercive adversaries come to play as a potential threat. For example, deniable encryption protects voters from being coerced during electronic elections [6,9]. It is also very useful to protect bidders in electronic auctions. Generally, deniable encryption is very important when a party is forced to act against his/her wish.

Our construction assumes the existence of the simple and efficient mediated-RSA (mRSA) [2] as the PKI in place. mRSA was invented as a method to achieve fast revocation in RSA PKI. mRSA involves a special entity, called the SEM (SEcurity Mediator), an on-line partially trusted server, to help signing or decrypting messages. The CA generates the private key $d$ corresponding to Bob's (the receiver's) public key $e$ and splits this private key into two pieces. One piece $\left(d_{S E M}\right)$ is delivered to the SEM and the other piece $\left(d_{B o b}\right)$ is delivered to Bob. The pair $(e, N)$ is the usual RSA public key. An important property to notice here is that Bob himself has no information neither about his full private key, nor about the factorization of the public modulus $\mathrm{N}$. This property is an excellent step toward achieving deniability since, a coercer will not ask Bob to reveal such unknown information. To decrypt a received cipher text, $C$, each party (Bob and SEM) performs his/her partial decryption on $C$; finally the partial decryptions are combined to recover the plaintext message $M$. To revoke Bob ability to sign or de- crypt messages, the CA instructs the SEM to stop issuing partial decryptions or signatures (spoken of as tokens) for Bob public key. At this instant, Bob's signature and/or decryption capabilities are revoked. The functionality is equivalent to (and indistinguishable from) standard RSA due to the fact that the splitting of the private key is transparent to the outside, i.e., to those who use the corresponding public key. Also, knowledge of a half-key cannot be used to derive the entire private key. Therefore, neither Bob nor the SEM can decrypt or sign a message without mutual consent.

As our PKI is established, we turn to discuss our tools. To complete the deniability service, we need an efficient protocol for (1-out-of- $n$ ) oblivious transfer $\left(\mathrm{OT}_{1}\right)$. Rabin proposed the concept of oblivious transfer (OT) in the cryptographic scenario. In this case the sender has only one secret bit $b$ and would like to have the receiver to get it with probability $1 / 2$, on the other hand, the receiver does not want the sender to know whether it gets $b$ or not. For $\mathrm{OT}_{1}$, the sender has two secrets $b_{1}$ and $b_{2}$, the receiver will get one of them at the receiver's choice.

The receiver does not want the sender to know which bit he chooses and the receiver must not know any information other than he chosen. Oblivious transfer is a fundamental primitive in many cryptographic applications and secure distributed computations and has many applications such as private information retrieval (PIR), fair electronic contract signing, oblivious secure computation, etc. Our proposed receiver-deniable public-key encryption scheme requires one invocation of $\mathrm{OT}_{1}$ between Bob and the SEM. Also we need a Secured mechanism [10] to make the OT protocol deniable.

The rest of the paper is organized as follows: Section 2 describes the related work. Section 3 gives our motivations and contributions. The underlying PKI and the oblivious transfer protocol are described in Section 4.

Section 5 states our assumptions and model. . The strong version is given in Section 6. Section 7 shows the techniques to transform deniability. The bandwidth is improved in Section 8 . Finally, the conclusions are given in Section 9.

\section{Related Work}

The work in [5] constructed a sender-deniable public- key encryption scheme based on trapdoor permutations. However, the scheme falls short of achieving an appropriate level of deniability, that is, to achieve a high deniability, the size of the cipher text corresponding to a one bit encryption is super-polynomial and hence inefficient. In the deniable public-key encryption scheme of [5], a one bit plaintext requires tn bits of cipher text where $t$ is the bit-length of elements in a translucent set $S t$ and $t=s+k$ for security parameters $n, s$ and $k$.

The scheme provides deniability of $4 / n$ and decryption error of $n 2-k$. Hence, to achieve a high level of deniability and a sufficiently low decryption error, the cipher text is super- polynomial and almost impractical [5]. Constructed two deniable public-key encryption schemes based on translucent sets, the first represents the building block for the second which they have called, the "Parity Scheme". The work in [5] also notified that in order to build a one-round scheme, different approaches are required. Also, [5] introduced techniques for the less challenging, deniable shared- key encryption and showed that the one-time-pad is a perfect deniable shared-key encryption.

Based on the sender-deniable public-key encryption, the work in [4] described a general multiparty computations allowing a set of players to compute a common function of their inputs with the ability to escape a coercion. In fact, deniable encryption has an impact on designing adaptively secure multiparty computations [4] since, the notion of deniability is stronger than the notion of non-committing encryption Schemes. 


\section{Motivations and Contributions}

In this Section we describe our motivations and contributions of the work in this paper.

\subsection{Motivations}

Deniable public-key encryption is a strong primitive, essential in all cryptographic protocols where a coercive adversary comes to play with high potential. Deniable public-key encryption realizes the "Receiptfreeness" attribute which is a very important attribute in electronic voting, electronic bidding and auctions. The schemes proposed in [5] fall short of achieving the desired level of deniability and correctness unless the size of the cipher text corresponding to a one bit encryption is super- polynomial. An appealing property in the mRSA PKI [2] is that the user himself has no information neither about his full private key, nor about the factorization of the public modulus $N$, consequently, a coercer will not ask the user for such unknown information.

\subsection{Contributions}

The contributions of this paper are to introduce an efficient receiver-deniable public-key encryption (RD-PKE) scheme. Our proposed scheme enjoys the following properties:

- It is a one-move scheme without any pre-encryption information required to be sent between the sender and the receiver prior to encryption.

- No pre-shared secret information is required between the sender and the receiver.

- Achieves a high level of deniability equivalent to the factorization of a large two-prime modulus.

- No deciphering errors.

- The bandwidth (cipher text bit-length) is significantly improved compared to previous constructions.

Efficiency, We reduce the required bandwidth (cipher- text bit-length) to, $2 \lg N$ bits for a single bit encryption, where $N$ is a two-prime RSA modulus. Moreover, this bandwidth can be efficiently improved, that is, $2 \lg N$ bits of cipher text allow about $\lg N-\delta$ bits of plaintext encryption where $\delta$ is a short randomizing string. At the same time, our scheme provides strong deniability (i.e. undetectable cheating) equivalent to the infeasibility to factor a sufficiently large two-prime modulus. Unlike the schemes of [5], our scheme produces no decryption errors and hence, more reliable. We introduce two versions of our RDPKE scheme, a weak version to declare our idea and security proofs, and then we show a simple modification to improve this weak version to be a strong RD-PKE scheme.

\subsection{Mediated RSA}

\section{Preliminaries}

Mediated RSA was invented as a simple method to achieve fast revocation in public-key cryptosystem. As usual, a trusted certificate authority (CA) sets up the RSA modulus $N$, the public exponent $e$ and the private exponent $d$ for the user. Next, instead of delivering $d$ to the user, the CA splits $d$ into two pieces $d_{S E M}$ and $d_{\text {user }}$ such that $d=d_{S E M}+d_{\text {user }} \bmod \phi(N)$ where $\phi(N)$ is the RSA Euler totient. Finally, the CA secretly delivers $d_{u s e r}$ to the user and $d_{S E M}$ to the SEM.

Encryption, For Alice to encrypt a message $\mathrm{M} \in Z_{N}$ to Bob, she uses Bob's public pair $(N, e)$ to compute the usual RSA cipher text $C=M^{e} \bmod N$ and sends $C$ to Bob.

Decryption, on the reception of $\mathrm{C}$ by Bob, the decryption process is as follows:

- Bob delivers $C$ to the SEM.

- If Bob's key is revoked, the SEM returns ERROR and aborts, else,

- The SEM computes her partial decryption $P D_{S E M}=\mathrm{C} d_{S E M \bmod N}$ and returns P $D_{S E M}$ to Bob. $\cdot$ Bob computes his partial decryption $P D_{B o b}=C d_{B o b}$

Mod N and extracts $M=P D_{S E M} P D_{B o b \bmod N \text {. }}$

It is important to notice that the SEM gains no information about the decrypted message $M$ [2].

\subsection{Oblivious Transfer}

Our proposed RD-PKE requires that Bob involves with the SEM in an OT1 invocation to get his encrypted bit. The main objective of the oblivious transfer protocols was to improve the efficiency and security of the protocols. The protocols of [18] have several appealing properties.

First, they prove efficiency over previous protocols, second, there are no number theoretic constrains on the strings to be obliviously transferred, third, the protocols have bandwidth-computation tradeoffs which make them suitable for variety of applications. The protocols of operate over a group $\mathrm{Z}_{q}$ of prime order, more precisely, $G_{q}$ is a subgroup of order q of $\mathrm{Z}_{*}$ where $\mathrm{p}$ is prime and $q / p-1$. Let g be a generator group and assume that the Diffie-Hellman assumption holds. In their OT1: The sender owns two strings $\mathrm{r}_{0}$ and $r_{1}$. He chooses a random element $U \in Z_{q}$ and publishes it. The chooser picks a random $1 \leq k \leq q$ and sets $p k \sigma=g k$ where $\in\{0$, $1\}$ is the chooser's choice. 
The chooser also computes $\mathrm{pk} 1-\sigma=U / p k \sigma$ and sends $\mathrm{pk} 0$ to the sender. The sender picks a random $\mathrm{R}$ and computes $\mathrm{gR}$ and $U R$, he also computes $\mathrm{pk}^{\mathrm{R}}$ and $\mathrm{pk}^{\mathrm{R}}=U^{R} / p k^{R}$. The sender computes $\mathrm{pk}_{1}-\sigma=U / p k \sigma$ and sends $\mathrm{pk}_{0}$ to the sender. The sender picks a random $\mathrm{R}$ and computes $\mathrm{gR}$ and $U R$, he also computes $\mathrm{pk}_{\mathrm{R}}$ and $\mathrm{pk}_{\mathrm{R}}=U^{R} / p k^{R}$. The sender The chooser selects a random $k$ and sets $p k \sigma=g k$ where $\sigma \in\{0, \ldots, n-1\}$ is his choice, it holds that $p k^{i}=U^{i} / p k^{0} \forall i=(1, \ldots, n-1)$. The chooser sends $\mathrm{pk}_{0}$ to the sender. The sender computes pkR as well as $p k^{R}$

\section{Assumptions and Model}

We define a receiver-deniable public-key encryption (RD-PKE) scheme as a scheme by which, the receiver is able to lie about the decrypted message to a coercer and hence, escape coercion. On one hand, the receiver is able to decrypt for the correct message, on the other hand, all the information held (or extractable) by the receiver when opened to a coercer, do not allow this coercer to verify the encrypted message and consequently, approaching the receiver becomes useless from the very beginning.

The participants in our scheme are the certificate authority (CA), the security mediator (SEM), the sender (Alice), the receiver (Bob) and the coercive adversary (coercer). As usual, the CA is assumed to be fully trusted by all participants. The SEM is a semi-trusted party in the sense that it follows the execution steps word for word but it is willing to learn any information that could be leaked during execution. Alice is assumed to be beyond the reach of any coercer while Bob is possibly coerced.

The coercer has the power to approach Bob coercing him to reveal the decrypted message, the decryption partial key and all the parameters he used during decryption.

This paper describes the scheme allowing one bit encryption at a time. The reader will notice that the scheme can be easily adapted to allow multiple bits encryption at a time. We assume that an mRSA PKI is already in place. Hence, the pair $(e, N)$ represents Bob's public key while $d_{B o b}$ (respectively $d_{S E M}$ ) are the pieces of Bob's private key $d$ held by Bob (respectively the SEM). Let $b t$ be the true bit to be encrypted by Alice to Bob. The scheme is described next.

Encryption. To encrypt the bit $b t$ to Bob, Alice proceeds as follows:

- Picks a $\lg N$ bits string $\mathrm{R} \in R Z N$. Let $r_{0} \ldots r_{n-1}$ be the binary representation of $R$.

- Scans the binary representation of $R$ for an index (Pointer) $i$ such that $r_{i}=b_{t}$

- Computes and sends the two encryptions, $C_{i}=i$

$\bmod N$ and $C_{R}=R^{e} \bmod N$ to Bob.

\section{Full Deniability}

In this Section we show how to achieve full deniability in our RD-PKE scheme, i.e., the scheme will be deniable even if the coercer is capable of eavesdropping the Alice-Bob channel and the SEM-Bob channel as well. The problem is that the OT protocol is not deniable and hence commits Bob to what he receives from the SEM. We benefit from the fact that the SEM and all its users are in the same domain (or System) this fact facilitates the sharing of a time-synchronous pseudo-random string between the SEM server and each user in its domain. Typical example is the OTPs (one time passwords) achieved via secure ID tokens (e.g. The well known and widely used tamper- resistance RSA-Secured tokens [10]). The SEM and the user in the SEM's domain share a pseudo-random string which is updated every 30 (or 60) seconds at both parties. It is important to notice that this pseudo-random string is synchronously shared based on internal clocks implemented at both parties, consequently, the update is performed offline without any communication, hence this pseudo-random string cannot be reached via eaves- dropping. Let $X(\tau)$ be the pseudo-random string shared between Bob and the SEM at any given time interval,

\section{Deniability Transformation}

$\mathrm{O}$ ur proposed scheme cannot withstand coercion of the sender, since a coerced sender is forced to reveal $R$ and the index $i$ which are verifiable by the coercer using the receiver's public key. A senderdeniable encryption is easily transformed to a receiver-deniable encryption and vice-versa. A sender-receiver deniable scheme requires $n$ intermediaries, $I_{1}, \ldots, I_{n}$, with at least one of them remains honest (un attacked). The sender chooses $n$ bits $b_{1}, \ldots, b_{n}$ such that $\mathrm{L} b_{i}=b_{t}$ and sends $b i$ to each $I_{i}$ using the sender deniable publickey encryption

\section{Bandwidth Improvement}

It is possible to further improve the bandwidth of our receiver-deniable public-key encryption scheme as follows: Let $M=\left\{M_{0}, \ldots, M_{m-1}\right\}$ be the set of all possible strings According to the plaintext message, Alice sets the indices

$I=i_{v-1}, \ldots, i_{0}$ where $i j$ points to $M i j$ in $R$. In this case, each index $i j$ is of ' bits where $<\lg \lg$ 
$N-\lg$ `. The maximum number of indices per encryption (i.e. Contained in $C_{I}$ ) is $v_{\max }{ }^{\prime} \lg N /$. Since each index points to a string of ' bits, then, the encryption pair $\left(C_{l}, C_{R}\right)$ encrypts about ' $\lg N /{ }^{\prime}=\lg N$ bits of plaintext. Hence, for a 1024 bits RSA modulus, a 2048 bits of cipher text encrypts $1024-\delta$ bits of plaintext where $\delta$ is the bit-length of $r$.

Finally, for each index, $i j$, Bob involves with the SEM in one invocation of OT1 oblivious transfer of strings to get $M i j$.

\section{Results and Discussion}

We proposed a scheme for receiver-deniable public-key encryption. Our scheme is based on mediated RSA PKI. Our scheme proves efficiency over that proposed in [5] in the sense of bandwidth, deniability and decipherability. The scheme can be transformed to a sender-deniable or a sender-receiver-deniable using the tricks of [5]. The complexity of the oblivious transfer protocol used in our RD-PKE was studied and improved in [11]. The reader may have noticed that, our proposed scheme is not restricted to RSA. Our scheme could be applied to any PKI with the mediated property. A final thing worth noting is that when our receiver-deniable scheme is transformed to a sender-deniable one, it is no more a one-move scheme. To construct a one-move sender-deniable scheme, other approaches must be invented. For example, one may consider the senderdeniable scheme in [12].

\section{References}

[1] M. Bellare, and P. Rogaway, "Random oracles are practical: a paradigm for designing efficient protocols," 1st Conference on Computer and Communications Security, pp.62-73, 1993.

[2] D. Boneh, X. Ding, G. Tsudik, and M. Wong, "A method for fast revocation of public key certificates and security capabilities," Proceedings of the 10 ${ }^{\text {th }}$ USENIX Security Symposium, pp. 297-308.

[3] C. Cachin, S. Micali, and M. Stadler, "Computationally private information retrieval with poly-logarithmic communication," Advances in Cryptography - Eurocrypt'99, pp. 402-414, 1999.

[4] R. Canetti, U. Feige, O. Goldreich, and M. Naor, "Adaptively secure multi-party computation," Proceedings 28th Annual ACM Symposium on Theory of Computing (STOC), pp. 639-648, 1996.

[5] R. Canetti, C. Dwork, M. Naor, and R. Ostrovsky, "Deniable encryption," Proceedings of the $17^{\text {th }}$ Annual international Cryptology Conference on Advances in Cryptology, pp. 90-104, Springer-Verlag, London, 1997.

[6] R. Cramer, R. Gennaro, and B. Schoenmakers, "A secure and optimally efficient multi-authority election scheme," Eurocrypt '97, pp. 103-118, 1997

[7] Y. Gertner, Y. Ishai, E. Kushilevitz, and T. Malkin, "Protecting data privacy in information retrieval schemes," Proceedings of $30^{\text {th }}$ Annual ACM Symposium on Theory of Computing, pp. 151-160, 1998.

[8] S. Goldwasser, and S. Micali, "Probabilistic encryption," Journal of Computer and System Sciences, vol. 28, no. 2, pp. 270-299, 1984.

[9] M. Hirt, and K. Sako, "Efficient receipt-free voting based on homomorphic encryption,” Eurocrypt '00, pp. 539-556, 2000.

[10] (http://www.rsa.com/rsalabs)

[11] M. H. Ibrahim, "Eliminating quadratic slowdown in two-prime RSA function sharing," Int. Journal of Network Security (IJNS), vol. 7, no. 1, pp.107-114, 2008

[12] M.H. Ibrahim, "A method for obtaining deniable public-key encryption," International Journal of Network Security (IJNS), to appear. 